\title{
Erratum to: Methods to account for tree-scale variability in soil and plant-related parameters in oil palm plantations
}

\author{
P. N. Nelson • M. J. Webb • M. Banabas • S. Nake • \\ I. Goodrick • J. Gordon • D. O'Grady • B. Dubos
}

Published online: 6 March 2014

(C) Springer International Publishing Switzerland 2014

\section{Erratum to: Plant Soil (2014) 374:459-471 \\ DOI 10.1007/s11104-013-1894-7}

The published online article unfortunately contained an error. Equation 19 should read as per below:

$H=S / 2 \cos 30^{\circ}$.

The online version of the original article can be found at http://dx. doi.org/10.1007/s11104-013-1894-7.

P. N. Nelson $(\bowtie) \cdot$ I. Goodrick · J. Gordon · D. O'Grady Centre for Tropical Environmental and Sustainability Science, James Cook University, PO Box 6811, Cairns, Qld 4870, Australia

e-mail: paul.nelson@jcu.edu.au

M. J. Webb

CSIRO Land \& Water, ATSIP Building, James Cook

University, Douglas, Qld 4811, Australia

M. Banabas

PNG Oil Palm Research Association, PO Box 28, Popondetta,

Oro Province, Papua New Guinea

S. Nake

PNG Oil Palm Research Association, PO Box 97, Kimbe,

West New Britain, Papua New Guinea

B. Dubos

CIRAD, Avenue Agropolis, 34398 Montpellier Cedex 5,

France 